Research Paper

\title{
Downregulation of miR-375 contributes to ERBB2- mediated VEGFA overexpression in esophageal cancer
}

\author{
Shuchang Ren ${ }^{1}$, Xiaohui Tann, Melinda Z. Fu ${ }^{1}$, Shuyang Ren ${ }^{1}$, Xiaoling $\mathrm{Wu}^{2}$, Tao $\mathrm{Chen}^{2}$, Patricia S. Latham³, \\ Paul Lin ${ }^{4}$, Yan-gao Man ${ }^{5}$ and Sidney W. Fu ${ }^{1 凶}$ \\ 1. Department of Medicine, Division of Genomic Medicine, and Department of Microbiology, Immunology and Tropical Medicine, The George Washington \\ University School of Medicine and Health Sciences, Washington, DC. \\ 2. Department of Medicine, Chengdu Military General Hospital, Chengdu, Sichuan, China. \\ 3. Department of Pathology, The George Washington University School of Medicine and Health Sciences, Washington, DC. \\ 4. Department of Surgery, The George Washington University School of Medicine and Health Sciences, Washington, DC. \\ 5. Department of Pathology, Hackensack Meridian Health-Hackensack, University Medical Center, Hackensack, NJ; the International Union for Difficult to \\ treat Diseases (IUDD), Silver Spring, MD.
}

$\square$ Corresponding author: Sidney W. Fu, M.D., Ph.D., Department of Medicine, Division of Genomic Medicine, The George Washington University School of Medicine and Health Sciences, 2300 Eye Street, N.W. Ross Hall 402C, Washington, DC 20037. Email: sfu@gwu.edu; Tel: 202-994-4767

(c) The author(s). This is an open access article distributed under the terms of the Creative Commons Attribution License (https://creativecommons.org/licenses/by/4.0/). See http://ivyspring.com/terms for full terms and conditions.

Received: 2021.06.13; Accepted: 2021.09.28; Published: 2021.10.20

\begin{abstract}
Esophageal cancer (EC) is a lethal cancer with an extremely aggressive nature and poor survival rate. However, the molecular mechanisms driving the occurrence and progression of EC are not well understood. MicroRNAs (miRNAs) are small RNA molecules that regulate the expression of protein-coding genes. miRNA-mediated gene regulation plays an important role in EC. By cross-referencing studies from NCBI, we found that microRNA-375 (miR-375) is one of the most frequently downregulated miRNAs in EC. We assessed expression of miR-375 in EC cell lines and primary EC tissues and their matched normal tissues. We found significant downregulation of miR-375 in both cell lines and EC tissues. Forced expression of miR-375 attenuated EC cell proliferation and invasion. Human epidermal growth factor receptor 2 (HER2, ERBB2), a known proto-oncogene, was identified here as one of the potential target genes of miR-375. Ectopic expression of miR-375 significantly suppressed the expression of ERBB2 and subsequently downregulated one of its target genes, vascular endothelial growth factor A (VEGFA), which is related to cancer invasion and metastasis. These findings suggest that miR-375 acts as a tumor suppressor by blocking the ERBB2/VEGFA pathway with the potential to modulate the occurrence and/ or progression of EC.
\end{abstract}

\section{Introduction}

Esophageal cancer (EC) is one of the deadliest cancers worldwide $[1,2]$. It consists of two common histologic types: esophageal squamous cell carcinoma (ESCC), which accounts for $80 \%$ of EC cases worldwide [3], and esophageal adenocarcinomas (EAC), which has a higher incidence in Western world [4]. Due to fact that most patients have distant metastases from EC at the time of diagnosis, the prognosis remains poor. The average five-year overall survival rates of the two type tumors are approximately 15\% [5]. However, the molecular mechanism of EC is not well understood. MicroRNAs (miRNAs) are small RNA molecules that regulate the expression of protein-coding genes by directly binding to target mRNAs in a sequence-specific manner. miRNAs are present in tissue, blood and other body fluids and have emerged as critical components of complex functional pathways involved in carcinogenesis. Specific miRNAs have been identified to be aberrantly expressed in ECs and found to correlate with diagnosis, prognosis and response to chemotherapy [6]. However, little is known about the cellular function of these differently expressed miRNAs.

By cross-referencing our RNASeq data (data not shown) with others' from NCBI, we found that miR-375 is one of the most frequently downregulated miRNAs in EC. The goal of this study was to 
investigate the mechanism of miR-375 dysregulation in EC.

\section{Materials and Methods}

\section{Cell lines}

ESCC cell lines, KYSE-70 and KYSE-180 were cultured in RPMI 1640 medium supplemented with $10 \%$ FBS and $1 \%$ penicillin and streptomycin antibiotics. EAC cell lines, FLO-1 and JHU-ad1, were cultured in Dulbecco's Modified Eagle Medium (DMEM) with 10\% FBS and 1\% penicillin and streptomycin antibiotics. These four cell lines were kindly provided by Dr. Stephen J. Meltzer (Johns Hopkins School of Medicine). The non-cancerous epithelial esophageal cell line HET-1A purchased from ATCC, was cultured in the base medium for this cell line (BEBM) along with BEGM kit (Catalog No. CC-3170, Lonza) with no GA-1000 (gentamycinamphotericin B mix). All the cell lines were incubated in a $37^{\circ} \mathrm{C}$ humidified incubator with $5 \% \mathrm{CO} 2$.

\section{Clinical samples and tissue microdissection}

The formalin-fixed, paraffin-embedded (FFPE) EC specimens were retrieved from the archive of the Chengdu Military General Hospital. None of the patients had received any chemotherapy or radiotherapy before sampling. The diagnosis of the archived esophageal cancer samples was confirmed by a second pathologist independently (Table 1). The FFPE tissues were microdissected into the following components, adjacent normal esophageal epithelium, dysplasia and carcinoma as described previously [7]. All sample collections were under the ethical standards of the institutional and national research committee and in agreement with the Helsinki Declaration and its later amendments or comparable ethical standards.

\section{RNA extraction and quantitative real-time reverse transcription-PCR (qRT-PCR)}

Total RNA from cultured cells and FFPE samples was isolated and quantitated as described previously [8]. miR-375-3p (Acc\#: MIMAT0000728) expression was assayed by qRT-PCR using the Taqman MiRNA Reverse Transcript Kit (Thermo Fisher Cat\# 4366596) with primer (5'- UUUGUUCGUUCGGCUCGCGUG A-3' Cat\# 4427975). The target gene ERBB2 (F: 5'-CAGTGCAGCACAGAGACTCA-3', R: 5'-CCG GTGCACACTCACTTTTG-3') and its downstream gene VEGFA (F: 5'-ACAAATGTGAATGCAGAC CAAA-3', R: 5'-ACCAACGTACACGCTCCAG-3') were analyzed using RT Real-Time ${ }^{\mathrm{TM}}$ SYBR Green (Bio-Rad Laboratories) as described previously [9].
Table 1. Clinical and pathologic parameters of FFPE samples from patient with EC.

\begin{tabular}{ll}
\hline Tissues parameters & Number \\
\hline Gender & \\
Male & 46 \\
Female & 11 \\
Age (Years) & \\
$31-60$ & 26 \\
$61-89$ & 31 \\
Tumor site & \\
Upper third & 17 \\
Middle third & 18 \\
Lower third & 13 \\
Esophagogastric junction & 8 \\
Unknown & 1 \\
Metastasis & \\
Non-metastasis & 18 \\
Metastasis & 39 \\
\# of Lymph nodes involved & \\
1 & 3 \\
2-5 & 11 \\
6-10 & 25 \\
Differentiation status & \\
Well & 13 \\
Moderate & 18 \\
Poor & 24 \\
Undifferentiated & 1 \\
Other & 1 \\
Depth of Invasion & \\
Mucosa & 4 \\
Submucosa & 5 \\
Muscularispropria & 18 \\
Serosa/full thickness & 30 \\
\hline
\end{tabular}

\section{Protein extraction and Western blotting}

Protein was extracted using the RIPA lysis buffer (ThermoFisher) according to the manufacturer's protocol. Briefly, proteins isolated from cells were separated electrophoretically. Equal amounts proteins $(50 \mu \mathrm{g})$ were run on $12 \%$ SDS-polyacrylamide gels and transferred onto nitrocellulose membrane. The following antibodies were used: anti-rabbit HER-2 (PA5-14635, ThermoFisher), anti-rabbit VEGF (PA5-16754, ThermoFisher), anti-mouse PhosphoAKT1 (44621G, ThermoFisher), anti-rabbit beta actin (PA1-183, ThermoFisher). Western blot analysis with chemiluminescent detection was performed as described [10].

\section{MiRNA and plasmid Transfection}

EC cells were seeded $\left(10^{6}\right.$ cells/well $)$ in a 12 -well plate in antibiotic-free medium for $24 \mathrm{~h}$ prior to transfection to achieve $80 \%$ confluency at the time of transfection. Transfection was conducted by the Lipofectamine RNAiMAX (Life Technologies, Cat\# 13778) delivery of miRNA precursors, including miR-375 mimic, inhibitors and their mock controls (ThermoFisher) using the Opti-MEM Reduced Serum Medium (Life Technologies). For rescue experiments, the pHAGE-ERBB2 plasmid containing full-length human ERBB2 cDNA without 3'UTR was purchased (Addgene, Cat\# 116734). The EC cell lines transfected 
with miR-375 mimic and inhibitor were then co-transfected with pHAGE/ERBB2 plasmid or empty pHAGE vector using the FuGENE reagent (Promega). The transfected cells were subjected to further analysis after $48 \mathrm{~h}$ post transfection.

\section{MTT assays}

For MTT assay, the cells transfected with miR-375 mimic, inhibitor or their corresponding mock controls were washed with 1xPBS. MTT working solution $(5 \mathrm{mg} / \mathrm{ml}$ stock MTT diluted in optiMEM to $0.5 \mathrm{mg} / \mathrm{ml}$ working solution) was added to each well and incubated at $37^{\circ} \mathrm{C}$ for $3 \mathrm{~h}$. MTT solution was then removed before adding $100 \mu \mathrm{l}$ of DMSO to each well for additional $30 \mathrm{~min}$ incubation. Color development was measured using a spectrophotometer at $490 \mathrm{~nm}$ on a plate reader (BIO-TEK Instruments) and quantified as per the manufacturer protocol (Promega, USA).

\section{Matrigel invasion assays}

EC Cell invasion capability was evaluated by Matrigel invasion assays using the BD BioCoat ${ }^{\mathrm{TM}}$ Matrigel $^{\mathrm{TM}}$ Invasion Chamber (BD Biosciences) according to the manufacturer's instructions as previously described [11]. Briefly, $500 \mu \mathrm{l}$ of warm $\left(37^{\circ} \mathrm{C}\right)$ serum-free DMEM medium was added to the upper and lower chambers and allowed to rehydrate for $2 \mathrm{~h}$ in a $37^{\circ} \mathrm{C}$ cell culture incubator, while $8 \times 10^{4}$ cells transfected by either miR-375 mimic or inhibitor with the mock controls for $24 \mathrm{~h}$, were seeded onto the top chamber of pre-wetted inserts. Cells were incubated in the Matrigel chamber in a $37{ }^{\circ} \mathrm{C}$ humidified incubator with $5 \% \mathrm{CO}_{2}$ for $48 \mathrm{~h}$. The non-invading cells were removed by scrubbing from the upper surface of the membrane with a cotton swab. The invasive cells present were fixed, stained with the Diff-Quick staining solution and counted (five microscope fields under the 10X len). Experiments were done in duplicates for each cell line twice. Cell counts were performed on five non-overlapping random fields for each chamber and four chambers were counted for each experimental using.

\section{Dual luciferase reporter assay}

For the luciferase reporter assay, $2 \times 10^{5}$ cells/well were plated in a 24-well plate. After $24 \mathrm{~h}$ incubation in a $37^{\circ} \mathrm{C}$ cell culture incubator, cells were co-transfected with $100 \mathrm{ng}$ of pEZX-ERBB2-3'UTR (wild type and mutant) expression clones inserted downstream of a secreted Gaussia luciferase (GLuc) reporter and $100 \mathrm{ng}$ of DNA with pEZX-miR-375 or the pEZX-MT scrambled control using the FuGENE Transfection Reagent (Promega). Luciferase activities were determined with the Secrete-PairTM Dual
Luminescence Assay Kit (Genecopoeia). GLuc luciferase activities were normalized to SEAP luciferase expression for each sample.

\section{Statistical analysis}

The difference of miR-375 expression in clinical samples was analyzed by the exact two-sided binomial test. Data were expressed as mean \pm standard error (S.E.). MTT assays between control and miR-375 mimic or inhibitor transfected groups were analyzed by Permutation tests. Matrigel assay between control and miR-375 or inhibitor transfected groups was analyzed using the student's t-test (two tailed). $P$ value less than 0.05 was considered statistical significance.

\section{Results}

\section{Decreased expression of miR-375 in EC cell lines and clinical samples}

To determine the expression of miR-375 in EC, we first measured the expression of miR-375 in EC cell lines by qRT-PCR. The expression levels of miR-375 in the four EC cell lines were lower than that in the non-cancerous epithelial esophageal cell line HET-1A (Fig. 1A). We then examined the expression of miR-375 in archival FFPE specimens, which were microdissected in pure populations of normal, dysplasia and tumor cells. Consistent with the findings in the cell lines, miR-375 was significantly decreased in 11 of 14 (78.6\%) ESCC compared to their adjacent areas of dysplasia and in 23 of 26 (88.5\%) ESCC compared to their adjacent normal tissues, respectively (Fig. 1D-F). These results suggest a high prevalence of downregulation of miR-375 during the progression of EC.

\section{miR-375 inhibits cell proliferation in EC}

After confirming the downregulation of miR-375 in EC, we sought to determine the functional role of miR-375 in EC. We firstly transfected miR-375 to EC cell lines and examined proliferation using MTT assays. Ectopic expression of miR-375 significantly inhibited the proliferation compared to the mock control in ESCC cell lines KYSE-70, EAC cell lines FLO-1 and JHU-ad1. Overexpression of miR-375 also inhibited the proliferation in ESCC cell line KYSE-180, although the change was not statistically significant. Conversely, transfection of miR-375 inhibitor significantly increased cell proliferation in ESCC cell line KYSE-70 and EAC cell line JHU-ad1 compared to transfected mock inhibitor. Transfection of miR-375 inhibitor also increased cell proliferation in ESCC cell line KYSE-180, although the change was not statistically significant (Fig. 2A). These results indicate a proliferative effect of miR-375 in EC cell lines. 


\section{miR-375 inhibits cell invasion in EC}

To test the impact of miR-375 on invasion, we performed Matrigel invasion assays in EC cell lines. Consistent with the effect of miR-375 on EC cell proliferation, a significantly decreased invasive capability was observed in miR-375-transfected ESCC cell lines KYSE-70, KYSE-180 and EAC cell line FLO-1, also in EAC cell line JHU-ad1 although the change was not statistically significant compared to the mock transfections. Conversely, transfection of miR-375 inhibitor significantly increased invasion capability compared to the mock inhibitor transfection in ESCC cell lines KYSE-180 and slightly increased invasion capability in KYSE-70 , FLO-1 and JHU-ad1, although the change was not statistically significant (Fig. 3).

miR-375 directly targets ERBB2 in EC

Using TargetScan and microrna.org

A

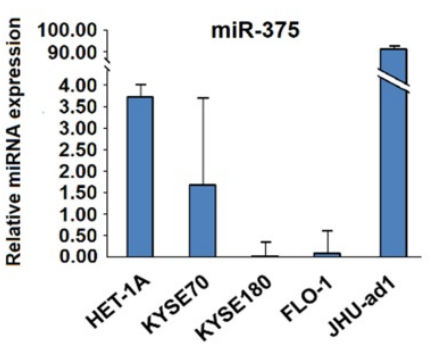

D

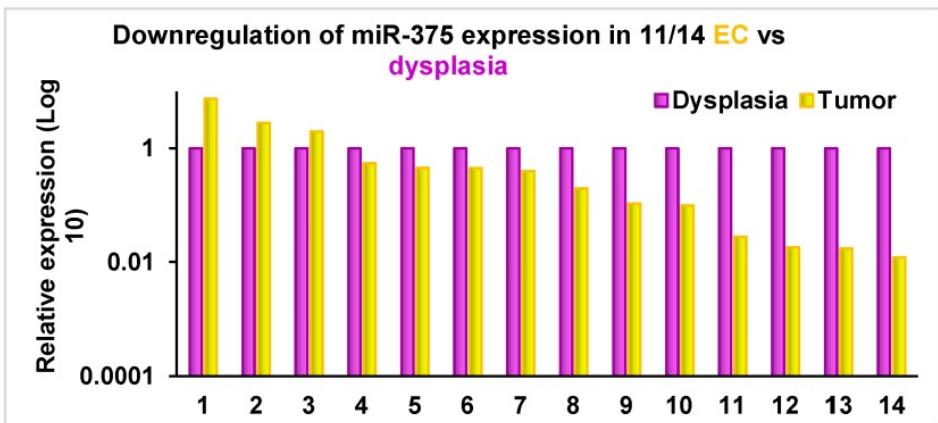

B

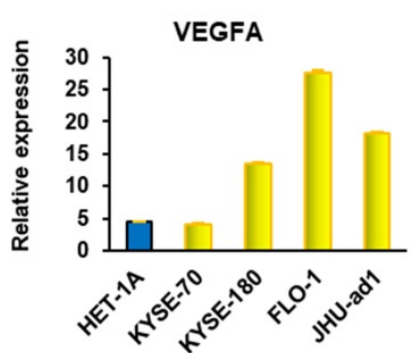

bioinformatics platforms, we identified a list of potential target genes of miR-375, including ERBB2. Since ERBB2 gene plays an important role in human malignancies and a high prevalence of ERBB2 expression has been demonstrated in EC [12], we focused on the regulatory role of miR-375 over ERBB2. We observed an inverse correlation between miR-375 and ERBB2 expression in EC cell lines (Fig. 1A and B). Furthermore, miR-375 transfection (Fig. 4A) resulted in significant ERBB2 downregulation compared to the mock transfection, while miR-375 inhibitor transfection restored the expression of ERBB2 compared to the inhibitor mock transfection in ESCC cell lines, KYSE-70, KYSE-180, and EAC cell line FLO-1, but not in JHU-ad1 (Fig. 4B). Our results suggest that miR-375 suppresses ERBB2 expression.

C

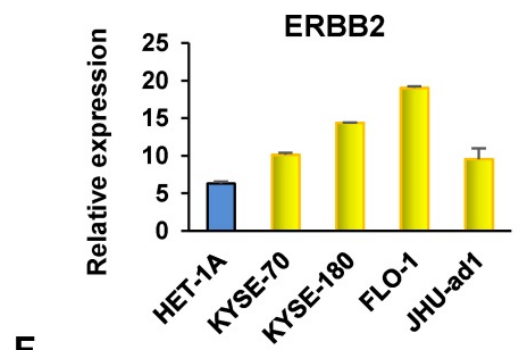

$\mathbf{F}$ miR-375 in EC vs dysplasia vs normal

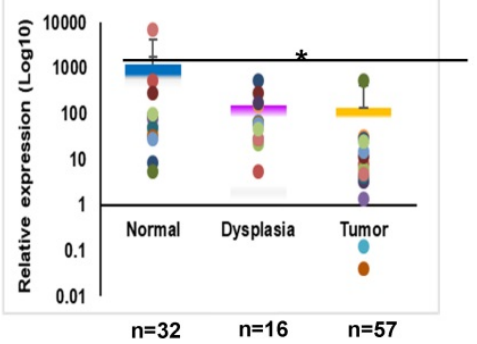

E

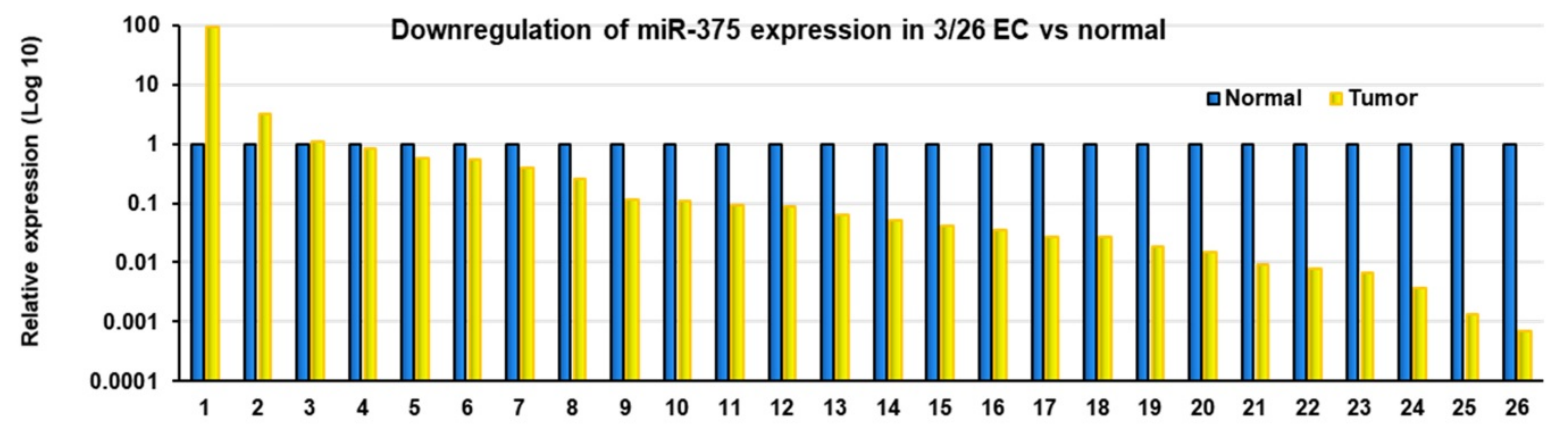

Figure 1. Expression of miR-375 and its target genes in EC. A. Expression of miR-375, ERBB2 and VEGFA in EC cell lines by qRT-PCR. Expression of miR-375 in EC cell lines are significantly decreased compared to HET-1A, a non-cancerous epithelial esophageal cell line. B and C. An inverse correlation between miR-375 and ERBB2/VEFGA expression pattern were observed in EC cell lines. D. Decreased miR-375 in pure population of tumor and dysplasia cells microdissected from FFPE tissue. The level of miR-375 expression was significantly decreased in 11 of 14 ESCC compared to their adjacent dysplasia. E. The level of miR-375 expression was significantly reduced in 23 of the 26 in ESCC compared with the adjacent normal tissues. F. Expression of miR-375 in normal, dysplasia and EC cells. Values represent the mean \pm S.D. from three independent experiments. (*p <0.05). 
A

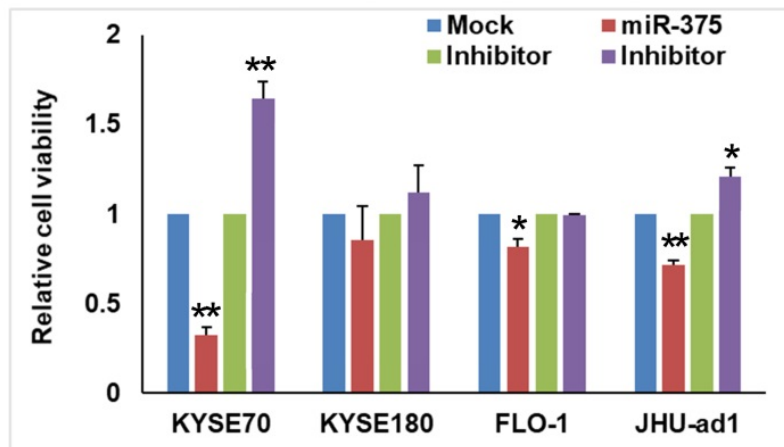

B

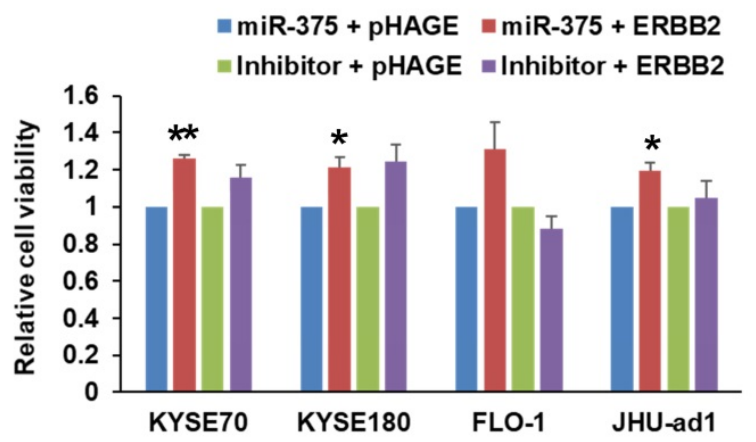

Figure 2. miR-375 inhibits cell proliferation that rescued by re-expression of ERBB2. A. miR-375 inhibits cell proliferation. The EC cell lines were transfected with mock control, miR-375 mimic, inhibitor control and miR-375 inhibitor; and cell viability was measured by MTT assay. Transfection of miR-375 mimic significantly reduced the proliferation compared to that of mock control in EC cell lines. Values represent the mean \pm S.D. from three independent experiments. (** $p<0.01$, *p $<0.05)$. B. Rescue experiment showed that the anti-proliferative effect of miR-375 is reversed by ERBB2 restoration. The PHAGE-ERBB2 cDNA expression vectors were transfected into EC cells after $48 \mathrm{~h}$ of miR-375 mimic or inhibitor transfection. The effect of ERBB2 restoration on cell proliferation in miR-375 transfected cell lines was measured by MTT. The cell proliferation was significantly increased after transfection of ERBB2 into miR-375 transfected or cells compared to that of PHAGE empty control in KYSE-70, FLO- 1 and JHU-ad I cells and KYSE-180, although the change was not statistically. Values represent the mean \pm S.D. for three independent experiments. $\left(* * \mathrm{p}<0.01, *_{\mathrm{p}}<0.05\right)$.

To confirm ERBB2 is a direct target of miR-375, we performed luciferase reporter assays by co-transfecting pEZX-MT05 vector containing HER2 3'UTR region with the miR-375 binding site (either wild type or mutant sequences), and pEZX-MT04 vector containing miR-375 (Fig. 4C) or scrambled control. The luciferase activities were significantly decreased in ESCC cell lines, KYSE-70 and KYSE-180 co-transfected with the pEZX-MT04 vectors containing miR-375 plus pEZX-MT05 vector containing ERBB2 3'UTR wild type sequence, compared to that co-transfection of either miR-375 plus 3'UTR mutant sequence or scrambled control plus ERBB2 3'UTR wild type one. Decreased luciferase activity was also observed in EAC cell lines, FLO-1 and JHU-ad1 co-transfected with miR-375 plus ERBB2 3'UTR wild type compared to that co-transfected with miR-375 plus scrambled control
(Fig. 4D). These results suggest that miR-375 directly regulates ERBB2 by binding to its 3'UTR in EC especially in ESCC.

\section{miR-375 suppresses ERBB2 mediated VEGF expression in ESCC}

The role of ERBB2 in angiogenesis has been well established and ERBB2 overexpression in human tumors is closely associated with increased VEGF [13, $14,15,16]$, which is well known to be related to invasion and metastasis of malignant tumors including EC [17, 18, 19]. Furthermore, ERBB2 has been implicated in the regulation of VEGF $[20,21]$. We observed an inverse correlation between miR-375 and ERBB2 expression as well as miR-375 and VEGFA expression in EC cell lines (Fig. 1A, B and C). As there are no predicted miR-375 binding sites at VEGFA mRNA 3'UTR, we hypothesize that miR-375 indirectly inhibits the expression of VEGF by suppressing ERBB2. In fact, ectopic transfection of miR-375 repressed not only ERBB2 but also VEGFA expression with significantly in ESCC but not in EAC cell lines. This result suggests that miR-375 suppresses ERBB2-mediated VEGF expression in ESCC.

\section{Tumor suppressive effects of miR-375 on ESCC cell lines were reversed by restoration of ERBB2 expression}

To verify whether miR-375 suppresses ESCC tumor growth by targeting ERBB2, a rescue experiment was conducted by transfecting pHAGEERBB2 plasmid containing full-length human ERBB2 cDNA into miR-375 transfected EC cell lines. As expected, the cell proliferation was significantly increased after transfecting pHAGE-ERBB2 into miR-375-transfected cell lines, KYSE-70, FLO-1 and JHU-ad1, compared to that of pHAGE empty vector control. For KYSE-180 cells, proliferation of the cells was also increased but not statistically significant (Fig. 2B). ERBB2 expression was reactivated in miR-375 inhibitor transfected EC cell lines, KYSE-70, KYSE-180 and JHU-ad1, although the change was not statistically significant (Fig. 2B).

To determine the effect of miR-375-mediated ERBB2 expression on cell invasion, we performed Matrigel invasion assay. Re-expression of ERBB2 enhanced the invasion capability compared to the pHAGE empty vector transfected one in EC cell lines (Fig. 3). In addition, Western blot results showed that re-expression of ERBB2 partially increased VEGF expression. These results suggest that miR-375 act as a tumor suppressor by inhibiting ERBB2 and/or ERBB2-mediated VEGF expression. 


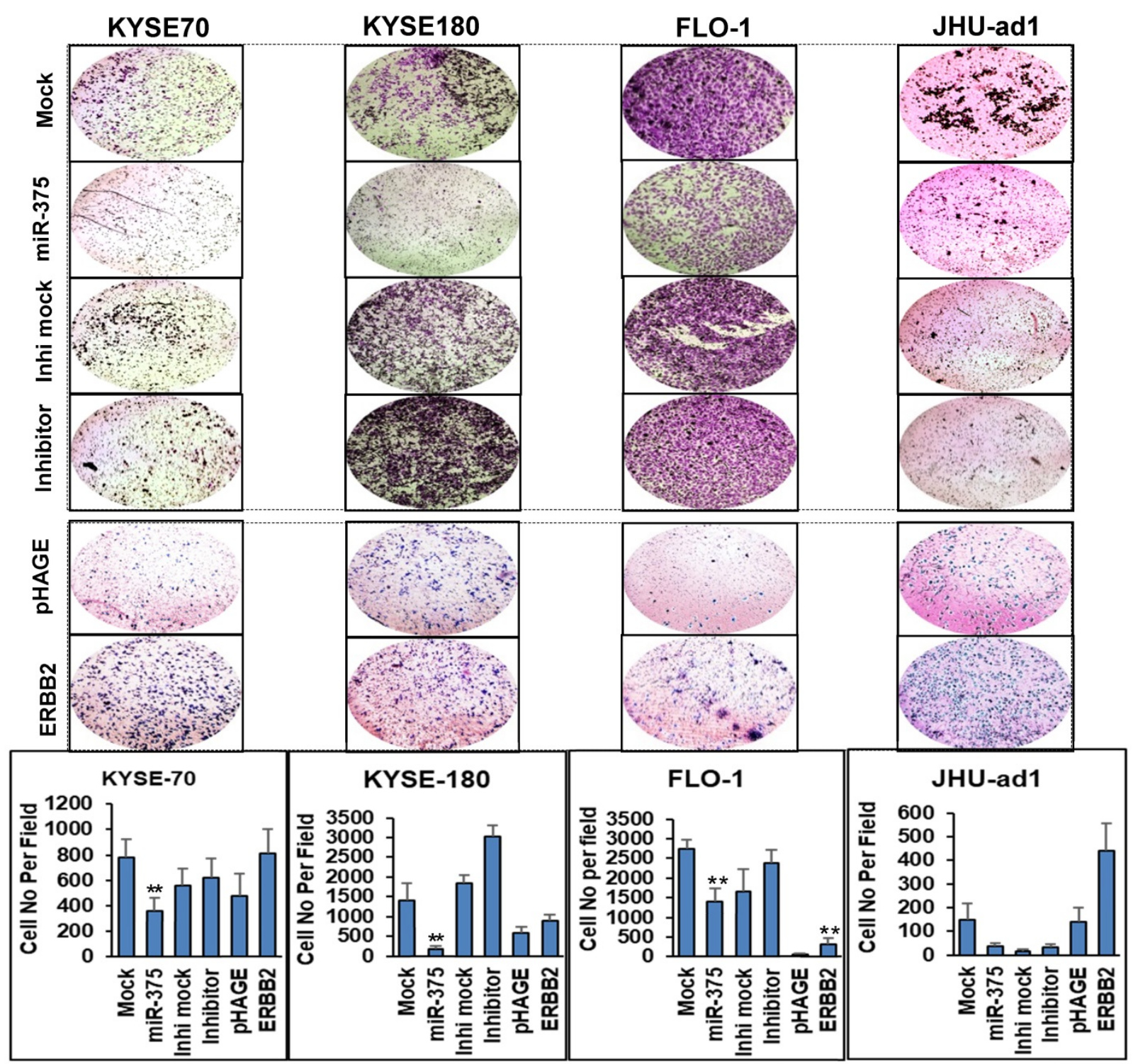

Figure 3. miR-375 inhibits invasive ability of EC cell lines that rescued by re-expression of ERBB2. Transwell assays were performed for the invasive activity of EC cells transfected with either miR-375 mimic or the inhibitor with their mock controls. Overexpression of miR-375 significantly reduced cell invasion in ESCC cell lines KYSE-70 and KYSE-180 and in EAC cell line FLO-1 but not significant in JHU-adl cells. Re-expression of ERBB2 resulted in significantly increased invasive ability compared to the pHAGE empty vector transfected one in EC cell lines. Invasive ability of the cells was displayed as a percentage of the absolute cell numbers (bottom). Results are displayed as mean data $\pm \mathrm{SE}$. $\left(* * \mathrm{p}<0.01,{ }^{*} \mathrm{p}<0.05\right)$. Five fields of unit area on each membrane or whole membrane were counted for cell numbers, and the experiments were repeated three times with triplicates.

\section{Discussion}

To date, dysregulation of miR-375 has been reported in a variety of cancers with different functions. Downregulated miR-375 was reported in the majority of cancers, such as oral squamous cell carcinoma [22], laryngeal squamous cell carcinoma [23], head and neck cancer [24], breast cancer [25], lung cancer [26], liver cancer [27], gastric carcinoma [28], colorectal cancer [29] and pancreatic cancer [30]. Upregulated miR-375 was mainly reported in urogenital carcinomas such as prostates cancer [31], ovarian cancer [32] and cervical cancer [33]. However, there are no reports about the regulation of miR-375 on ERBB2/VEGF pathway in EC to the best of our knowledge. We found decreased miR-375 expression in EC tissue as well as pre-cancerous dysplasia. miR-375 is located on chromosome $2 q 35$ [34], which is often deleted in EC [35], suggesting that the downregulation of miR-375 may be due to genomic DNA deletion in human EC. If so, it is possible that decreased levels of miR-375 may serve as a potential diagnostic marker for the early detection of EC.

ERBB2 proto-oncogene is a type I transmembrane tyrosine kinase growth factor receptor that plays a key role in the processes of tumor cell proliferation, differentiation and growth [36]. Overexpression of HER2 in human tumor cells is closely associated with increased VEGF expression [13]. 

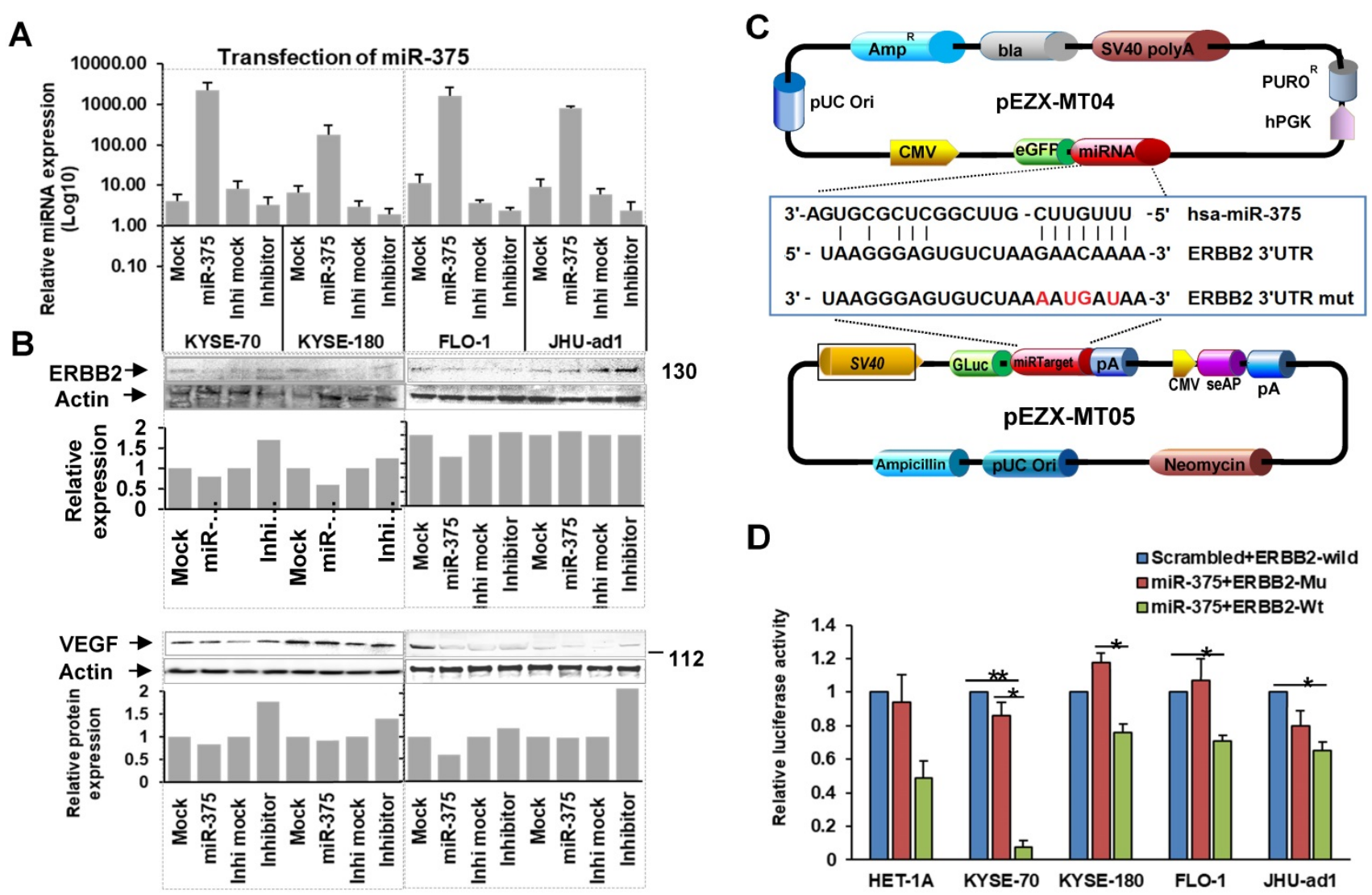

Figure 4. miR-375 regulates ERBB2 and VEGFA in EC. A. Expression of miR-375 after transfection. B. Forced expression of miR-375 in EC cell lines resulted in decreased ERBB2 and VEGFA expression. C. Map of the plasmids pEZX-MT04 containing miR-375, and pEZX-MT05 containing 3'-UTR of ERBB2 to illustrate the binding site of miR-375 at the 3'-UTR of ERBB2, and its mutant control sequence. D. Dual luciferase reporter assay. Co-transfection with pEZX-miR-375 and pEZX-ERBB2 3' UTR wild type significantly decreased the luciferase activities compared to that with pEZX-miR-375 and ERBB2 3' UTR mutant/miR-375 scrambled control and ERBB2 3' UTR wild type sequence in ESCC cell lines. The data were reported as mean \pm S.D. from three independent experiments $\left(* * p<0.01,{ }^{*} p<0.05\right)$

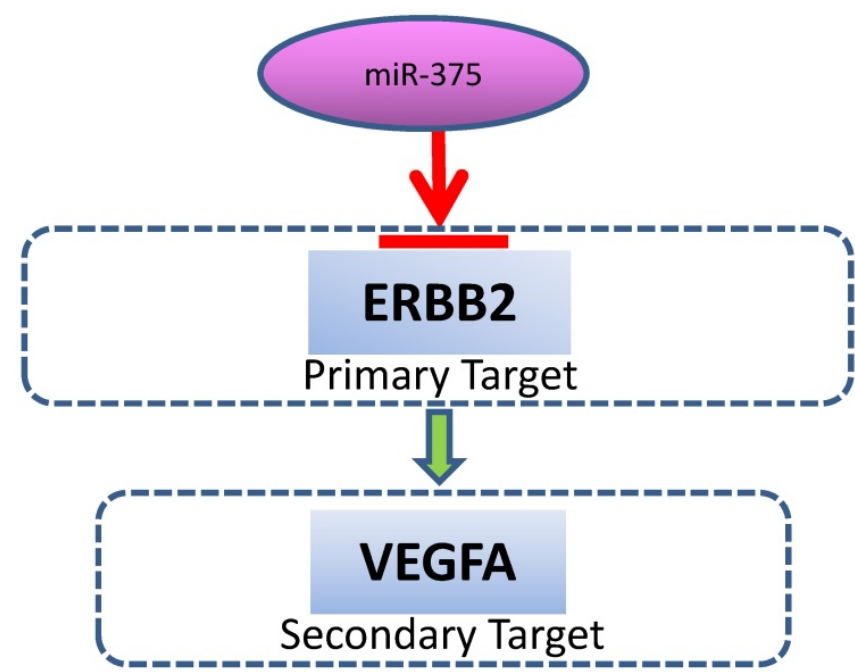

Figure 5. A schematic model for the regulation of miR-375. miR-375 directly targets ERBB2, resulting in VEGF downregulation.

Overexpression and/or amplification of ERBB2 and VEGF have been found in a variety of human cancers, including EC [12, 18, 19, 37-45]. ERBB2 and VEGF are located on chromosome 17q12 and 6p21.1, respectively. As both regions are frequently amplified in EC $[46,47]$, ERBB2 and VEGF overexpression can be therapeutic targets for GI cancer. In fact, combined blockade of both HER2 and VEGF exerts synergistic tumor growth inhibition in gastroesophageal cancer [48]. In this study, we found that decreased miR-375 is often associated increased ERBB2/VEGF expression in EC. Our data demonstrate that ERBB2 is a direct target of miR-375 and VEGFA can be indirectly targeted by miR-375, implying that frequent overexpression of ERBB2 and VEGF may be, at least in part, due to downregulation of miR-375 in the development of EC. We further demonstrate that, overexpression of miR-375 significantly suppressed proliferation and invasion in EC cells. These findings suggest that miR-375 is potentially an important tumor suppressor in the initiation and/or progression of EC by targeting ERBB2/VEGF. The miR-375/ ERBB2/VEGF axis might serve as a signature for both diagnostic and therapeutic target in EC. Both drugs that specifically target ERBB2 and VEGF have been shown to confer clinical benefit $[49,50]$. The fact that miR-375 targets both ERBB2 and VEGF suggests that it may serve as an upstream target for EC treatment.

Esophageal cancer is composed of two distinct histological subtypes, ESCC and EAC. The two types of EC arise from different cell populations, with ESCC 
from squamous epithelium and EAC from intestinal metaplastic epithelial cells or Barrett's esophagus. Although miR-375 was considered as a tumor suppressor in the majority of cancers, the role of miR-375 in tumorigenesis is still controversial. For example, miR-375 expression was significantly up-regulated in adenocarcinoma and small cell lung carcinoma but down-regulated in squamous cell carcinoma [26]. In our study, the tumor suppressor function of miR-375 was not significantly different between ESCC and EAC, suggesting a broadspectrum tumor suppressor effect in EC.

In conclusion, this study provides evidence that miR-375 is a tumor suppressor which is frequently suppressed in both EC and precancerous lesions. Decreased miR-375 is associated with overexpression of both ERBB2 and VEGF oncogene genes. These findings suggest that miR-375 may serve as a novel target for EC treatment.

\section{Acknowledgements}

The study was supported by the Virginia Gray Fund (to SWF), and the Elaine H. Snyder Cancer Research Award (to SWF).

\section{Competing Interests}

The authors have declared that no competing interest exists.

\section{References}

1. Esophageal cancer: epidemiology, pathogenesis and prevention. Nat Clin Pract Gastroenterol Hepatol. 2008; 5: 517-26.

2. Ferlay J, Shin HR, Bray F, Forman D, Mathers C, Parkin DM. Estimates of worldwide burden of cancer in 2008: GLOBOCAN 2008. Int J Cancer. 2010; 127: 2893-917.

3. Taylor PR, Abnet CC, Dawsey SM. Squamous Dysplasia-The Precursor Lesion for Esophageal Squamous Cell Carcinoma. Cancer Epidemiology Biomarkers \& Prevention. 2013; 22: 540-52.

4. Bandla S, Pennathur A, Luketich JD, Beer DG, Lin L, Bass AJ, et al. Comparative genomics of esophageal adenocarcinoma and squamous cell carcinoma. Ann Thorac Surg. 2012; 93: 1101-6.

5. Portale G, Hagen JA, Peters JH, Chan LS, DeMeester SR, Gandamihardja TA, et al. Modern 5-year survival of resectable esophageal adenocarcinoma: single institution experience with 263 patients. J Am Coll Surg. 2006; 202: 588-96; discussion 96-8.

6. Tan X, Ren S, Fu MZ, Ren S, Yang C, Wu X, et al. microRNA-196b promotes esophageal squamous cell carcinogenesis and chemoradioresistance by inhibiting EPHA7, thereby restoring EPHA2 activity. Am J Cancer Res. 2021; 11: 3594-610.

7. Chen L, Li Y, Fu Y, Peng J, Mo MH, Stamatakos M, et al. Role of deregulated microRNAs in breast cancer progression using FFPE tissue. PLoS One. 2013; 8: e54213.

8. Tan X, Fu Y, Chen L, Lee W, Lai Y, Rezaei K, et al. miR-671-5p inhibits epithelial-to-mesenchymal transition by downregulating FOXM1 expression in breast cancer. Oncotarget. 2015; 6

9. Tan X, Peng J, Fu Y, An S, Rezaei K, Tabbara S, et al. miR-638 mediated regulation of BRCA1 affects DNA repair and sensitivity to UV and cisplatin in triple-negative breast cancer. Breast Cancer Res. 2014; 16: 435.

10. Tan X, Anzick SL, Khan SG, Ueda T, Stone G, Digiovanna JJ, et al. Chimeric negative regulation of p14ARF and TBX1 by a $t(9 ; 22)$ translocation associated with melanoma, deafness, and DNA repair deficiency. Hum Mutat. 2013; 34: 1250-9.

11. Fu Y, Lian Y, Kim KS, Zhang L, Hindle AK, Brody F, et al. BP1 Homeoprotein Enhances Metastatic Potential in ER-negative Breast Cancer. J Cancer. 2010; 1: 54-62.

12. Gowryshankar A, Nagaraja V, Eslick GD. HER2 status in Barrett's esophagus \& esophageal cancer: a meta analysis. J Gastrointest Oncol. 2014; 5: 25-35.
13. Kumar R. The role of HER2 in angiogenesis. Seminars in Oncology. 2001; 28: 27-32.

14. Yang W, Klos K, Yang Y, Smith TL, Shi D, Yu D. ErbB2 overexpression correlates with increased expression of vascular endothelial growth factors $\mathrm{A}$, C, and D in human breast carcinoma. Cancer. 2002; 94: 2855-61.

15. Laughner E, Taghavi P, Chiles K, Mahon PC, Semenza GL. HER2 (neu) signaling increases the rate of hypoxia-inducible factor 1alpha (HIF-1alpha) synthesis: novel mechanism for HIF-1-mediated vascular endothelial growth factor expression. Mol Cell Biol. 2001; 21: 3995-4004.

16. Wen XF, Yang G, Mao W, Thornton A, Liu J, Bast RC, Jr., et al. HER2 signaling modulates the equilibrium between pro- and antiangiogenic factors via distinct pathways: implications for HER2-targeted antibody therapy. Oncogene. 2006; 25: 6986-96.

17. Wei W, Wang Y, Yu X, Ye L, Jiang Y, Cheng Y. Expression of TP53, BCL-2, and VEGFA Genes in Esophagus Carcinoma and its Biological Significance. Med Sci Monit. 2015; 21: 3016-22.

18. Kleespies A, Guba M, Jauch KW, Bruns CJ. Vascular endothelial growth factor in esophageal cancer. J Surg Oncol. 2004; 87: 95-104.

19. Kleespies A, Bruns CJ, Jauch KW. Clinical significance of VEGF-A, -C and -D expression in esophageal malignancies. Onkologie. 2005; 28: 281-8.

20. Klos KS, Wyszomierski SL, Sun M, Tan M, Zhou X, Li P, et al. ErbB2 increases vascular endothelial growth factor protein synthesis via activation of mammalian target of rapamycin/p70S6K leading to increased angiogenesis and spontaneous metastasis of human breast cancer cells. Cancer Res. 2006; 66: 2028-37.

21. Yen L, Benlimame N, Nie ZR, Xiao D, Wang T, Al Moustafa AE, et al. Differential regulation of tumor angiogenesis by distinct ErbB homo- and heterodimers. Mol Biol Cell. 2002; 13: 4029-44.

22. Tsai SC, Huang SF, Chiang JH, Chen YF, Huang CC, Tsai MH, et al. The differential regulation of microRNAs is associated with oral cancer. Oncol Rep. 2017; 38: 1613-20.

23. Chang K, Wei Z, Cao H. miR-375-3p inhibits the progression of laryngeal squamous cell carcinoma by targeting hepatocyte nuclear factor-1beta. Oncol Lett. 2020; 20: 80 .

24. Hui $\mathrm{AB}$, Bruce JP, Alajez NM, Shi W, Yue S, Perez-Ordonez B, et al. Significance of dysregulated metadherin and microRNA-375 in head and neck cancer. Clin Cancer Res. 2011; 17: 7539-50.

25. de Souza Rocha Simonini P, Breiling A, Gupta N, Malekpour M, Youns M, Omranipour R, et al. Epigenetically deregulated microRNA-375 is involved in a positive feedback loop with estrogen receptor alpha in breast cancer cells. Cancer Res. 2010; 70: 9175-84.

26. Jin Y, Liu Y, Zhang J, Huang W, Jiang H, Hou Y, et al. The Expression of miR-375 Is Associated with Carcinogenesis in Three Subtypes of Lung Cancer. PLoS One. 2015; 10: e0144187.

27. Li L, Jia L, Ding Y. Upregulation of miR-375 inhibits human liver cancer cell growth by modulating cell proliferation and apoptosis via targeting ErbB2. Oncol Lett. 2018; 16: 3319-26.

28. Tsukamoto $Y$, Nakada C, Noguchi T, Tanigawa M, Nguyen LT, Uchida T, et al. MicroRNA-375 is downregulated in gastric carcinomas and regulates cell survival by targeting PDK1 and 14-3-3zeta. Cancer Res. 2010; 70: 2339-49.

29. Xu F, Ye ML, Zhang YP, Li WJ, Li MT, Wang HZ, et al. MicroRNA-375-3p enhances chemosensitivity to 5 -fluorouracil by targeting thymidylate synthase in colorectal cancer. Cancer Sci. 2020; 111: 1528-41.

30. Qu K, Zhang X, Lin T, Liu T, Wang Z, Liu S, et al. Circulating miRNA-21-5p as a diagnostic biomarker for pancreatic cancer: evidence from comprehensive miRNA expression profiling analysis and clinical validation. Sci Rep. 2017; 7: 1692.

31. He S, Shi J, Mao J, Luo X, Liu W, Liu R, et al. The expression of miR-375 in prostate cancer: A study based on GEO, TCGA data and bioinformatics analysis. Pathol Res Pract. 2019; 215: 152375

32. Su YY, Sun L, Guo ZR, Li JC, Bai TT, Cai XX, et al. Upregulated expression of serum exosomal miR-375 and miR-1307 enhance the diagnostic power of CA125 for ovarian cancer. J Ovarian Res. 2019; $12: 6$.

33. Shen Y, Zhou J, Li Y, Ye F, Wan X, Lu W, et al. miR-375 mediated acquired chemo-resistance in cervical cancer by facilitating EMT. PLoS One. 2014; 9: e109299.

34. Baroukh NN, Van Obberghen E. Function of microRNA-375 and microRNA-124a in pancreas and brain. FEBS J. 2009; 276: 6509-21.

35. Liu X, Zhang M, Ying S, Zhang C, Lin R, Zheng J, et al. Genetic Alterations in Esophageal Tissues From Squamous Dysplasia to Carcinoma. Gastroenterology. 2017; 153: 166-77.

36. Fichter CD, Gudernatsch V, Przypadlo CM, Follo M, Schmidt G, Werner M, et al. ErbB targeting inhibitors repress cell migration of esophageal squamous cell carcinoma and adenocarcinoma cells by distinct signaling pathways. J Mol Med (Berl). 2014; 92: 1209-23.

37. Plum PS, Gebauer F, Kramer M, Alakus H, Berlth F, Chon SH, et al. HER2/neu (ERBB2) expression and gene amplification correlates with better survival in esophageal adenocarcinoma. BMC Cancer. 2019; 19: 38.

38. Gerson JN, Skariah S, Denlinger CS, Astsaturov I. Perspectives of HER2-targeting in gastric and esophageal cancer. Expert Opin Investig Drugs. 2017; 26: 531-40.

39. Konig AM, Reeh M, Dancau AM, Rathjens M, Gros S, Uzunoglu FG, et al. Concordance of HER2 status in primary tumour and lymph node metastases in patients with esophageal carcinoma. Anticancer Res. 2013; 33: 4975-82. 
40. Taghizadeh Kermani A, Vakili R, Dadkhah S, Jafarian AH, Bagheri R. HER-2/neu Overxpression in Esophageal Squamous Cell Carcinoma (ESCC) and Its Correlation with Patient's Clinicopathological Features. Iranian Journal of Cancer Prevention. 2016; In Press.

41. Kashyap MK, Abdel-Rahman O. Expression, regulation and targeting of receptor tyrosine kinases in esophageal squamous cell carcinoma. Mol Cancer. 2018; 17: 54 .

42. Hardwick RH, Barham CP, Ozua P, Newcomb PV, Savage P, Powell R, et al. Immunohistochemical detection of p53 and c-erbB-2 in oesophageal carcinoma; no correlation with prognosis. European Journal of Surgical Oncology (EJSO). 1997; 23: 30-5.

43. Mimura K, Kono K, Hanawa M, Mitsui F, Sugai H, Miyagawa N, et al. Frequencies of HER-2/neu expression and gene amplification in patients with oesophageal squamous cell carcinoma. Br J Cancer. 2005; 92: 1253-60.

44. Stoecklein NH, Hosch SB, Bezler M, Stern F, Hartmann CH, Vay C, et al. Direct genetic analysis of single disseminated cancer cells for prediction of outcome and therapy selection in esophageal cancer. Cancer Cell. 2008; 13: 441-53.

45. Zhan N, Dong WG, Tang YF, Wang ZS, Xiong CL. Analysis of HER2 gene amplification and protein expression in esophageal squamous cell carcinoma. Med Oncol. 2012; 29: 933-40.

46. Sawada G, Niida A, Hirata $H$, Komatsu H, Uchi R, Shimamura T, et al. An Integrative Analysis to Identify Driver Genes in Esophageal Squamous Cell Carcinoma. PLoS One. 2015; 10: e0139808.

47. Maqani N, Belkhiri A, Moskaluk C, Knuutila S, Dar AA, El-Rifai W. Molecular dissection of 17q12 amplicon in upper gastrointestinal adenocarcinomas. Mol Cancer Res. 2006; 4: 449-55.

48. Singh R, Kim WJ, Kim PH, Hong HJ. Combined blockade of HER2 and VEGF exerts greater growth inhibition of HER2-overexpressing gastric cancer xenografts than individual blockade. Exp Mol Med. 2013; 45: e52.

49. Davidson M, Starling N. Trastuzumab in the management of gastroesophageal cancer: patient selection and perspectives. Onco Targets Ther. 2016; 9: 7235-45.

50. Young K, Smyth E, Chau I. Ramucirumab for advanced gastric cancer or gastro-oesophageal junction adenocarcinoma. Therap Adv Gastroenterol. 2015; 8: 373-83. 